\title{
Numerical study of resonant spin relaxation in quasi-one-dimensional channels
}

\author{
S. Lüscher, ${ }^{1}$ S. M. Frolov, ${ }^{1,2}$ and J. A. Folk ${ }^{1}$ \\ ${ }^{1}$ Department of Physics and Astronomy, University of British Columbia, Vancouver, British Columbia, Canada V6T 1 Z4 \\ ${ }^{2}$ Kavli Institute of Nanoscience, Delft University of Technology, 2600 GA Delft, The Netherlands \\ (Received 1 April 2010; revised manuscript received 13 August 2010; published 7 September 2010)
}

\begin{abstract}
Recent experiments demonstrate that a ballistic version of spin resonance, mediated by spin-orbit interaction, can be induced in narrow channels of a high-mobility GaAs two-dimensional electron gas by matching the spin-precession frequency with the frequency of bouncing trajectories in the channel. Contrary to the typical suppression of D'yakonov-Perel' spin relaxation in confined geometries, the spin-relaxation rate increases by orders of magnitude on resonance. Here, we present Monte Carlo simulations of this effect to explore the roles of varying degrees of disorder and strength of spin-orbit interaction. Quantitative spin-orbit parameters can be extracted by comparing simulations of this type with experimental measurements of ballistic spin resonance, guiding the development of future spintronic devices.
\end{abstract}

DOI: 10.1103/PhysRevB.82.115304

PACS number(s): 73.23.-b, 72.25.Rb, 72.25.Dc, 75.40.Mg

\section{INTRODUCTION}

Spin-orbit interaction, which couples an electron's spin degree of freedom to its momentum, is the primary source of spin relaxation for free carriers in semiconductors. ${ }^{1,2}$ At the same time, it offers the potential to control the spin orientation of those carriers without the need for conventional highfrequency resonance techniques. ${ }^{3-5}$ Controlling carrier spins using spin-orbit interaction requires the ability to tune its effect with external parameters such as a magnetic field or voltages on electrostatic gates. In the Datta-Das spintransistor concept, for example, the spins of carriers in a two-dimensional (2D) quantum well rotate in response to a spin-orbit interaction whose strength can be tuned by a gate. ${ }^{4,6}$

Another way that electrostatic gates can tune the effects of spin-orbit interaction in a quantum well is by defining the lateral confinement geometry of a spintronic device. Recent experiments have shown that bouncing trajectories in gatedefined channels of high-mobility GaAs 2D electron gas (2DEG), in an external magnetic field, lead to rapid spin relaxation through a process we refer to as ballistic spin resonance (BSR). ${ }^{5}$ On resonance the effect of spin-orbit interaction is amplified by matching the Larmor precession frequency with the bouncing frequency, which depends on the gate-defined channel width. Although the mechanism of BSR is straightforward, it is not obvious that the effect should be visible for realistic parameters in a practical device.

In this Brief Report, semiclassical Monte Carlo simulations of spin dynamics are used to test the resilience of BSR over a wide range of device parameters. The simulation models varying degrees and types of disorder, confinement potential from the electrostatic gates, and lack of perfect specularity on scattering off the channel walls. A range of spinorbit interaction strengths are explored in the simulation, including terms both linear and cubic in the momentum. We restrict our attention to electron-doped GaAs 2DEGs at low temperature, where BSR was observed experimentally. ${ }^{5}$ BSR is found to be robust over a wide range of experimentally accessible parameters and not to depend sensitively on specific model of disorder.

\section{SPIN-ORBIT INTERACTION AND D'YAKONOV- PEREL' MECHANISM}

Spin-orbit interaction in III-V semiconductor quantum wells is characterized by Rashba $(\alpha)$ and Dresselhaus ( $\beta$ and $\gamma)$ terms, due, respectively, to structural and bulk crystal inversion asymmetry. ${ }^{2}$ Including both types of spin-orbit interaction, the spin-orbit Hamiltonian for a 2D quantum well grown along the [001] crystal axis is

$$
\begin{aligned}
H_{s o}= & \alpha\left(k_{010} \sigma_{100}-k_{100} \sigma_{010}\right)+\beta\left(k_{100} \sigma_{100}-k_{010} \sigma_{010}\right) \\
& +\gamma\left(k_{010}^{2} k_{100} \sigma_{100}-k_{100}^{2} k_{010} \sigma_{010}\right),
\end{aligned}
$$

where $k_{100}$ is the component of the Fermi wave vector along the [100] crystal axis and $\sigma_{100}$ is the Pauli spin operator along the [100] axis. We note that the sign convention adopted for this Hamiltonian gives $\beta \approx-\gamma\left\langle k_{001}^{2}\right\rangle,{ }^{7,8}$

The linear-in- $k$ terms in the Hamiltonian become simpler when described along [110] and [110] axes. For the rest of this paper, the [110] axis is referred to as the $x$ axis; the [ $\overline{1} 10]$ axis is referred to as the $y$ axis. $H_{s o}$ can be interpreted in terms of a momentum-dependent effective Zeeman field, $\vec{B}_{s o}$, which takes the following form when expressed along $x$ and $y$ axes:

$$
\vec{B}_{s o}=\frac{2}{g \mu_{B}}\left[(\alpha-\beta) k_{y} \hat{x}-(\alpha+\beta) k_{x} \hat{y}\right]+O\left(k^{3}\right) .
$$

This effective field corresponds to a spin-orbit precession time $\tau_{s o}=\left|\hbar / g \mu_{B} B_{s o}\right|$, where $g=-0.44$ is the Landé $g$ factor in GaAs. Equation (2) illustrates that spin-orbit interaction is anisotropic when $\alpha$ and $\beta$ are of the same order, which is the case in GaAs triangular wells. ${ }^{9}$ In the simulations presented here, the values of $\alpha$ and $\beta$ are varied widely and change sign, meaning that no fixed bulk spin-orbit anisotropy is assumed.

In principle, spins precess coherently according to $H_{s o}$ as they travel through the crystal. At a practical level, however, $H_{s o}$ gives rise to spin relaxation in any real conductor due to momentum scattering. Electron spins precess around an effective magnetic field that changes, as the momentum changes, at each scattering event. An ensemble of polarized 

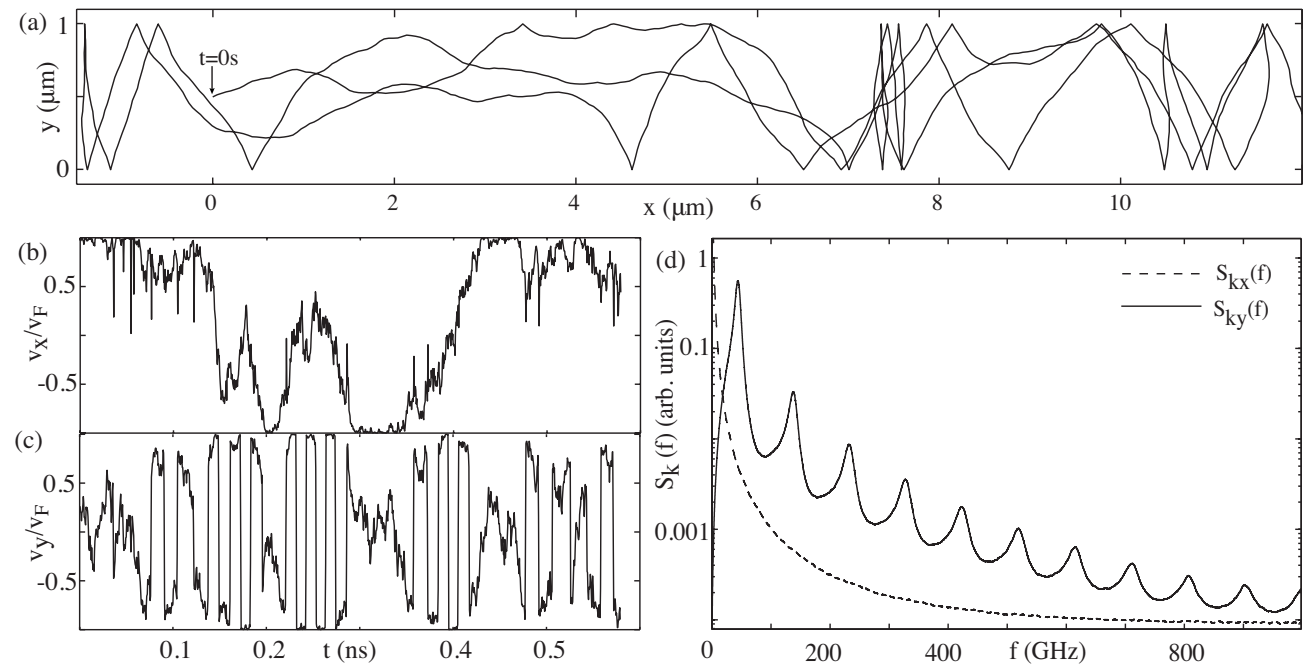

FIG. 1. (a) An example of a trajectory segment in a $1-\mu \mathrm{m}$-wide wire with $\lambda=10 \mu \mathrm{m}$ due to small angle scattering. Because the channel width is much less than the mean free path, the time dependence of the $x$ and $y$ components of the velocity, $v_{x}$ and $v_{y}$, respectively, are qualitatively different [panels (b) and (c)]. (d) Power spectral densities show the square-wave nature of $k_{y} \propto v_{y}$ but no characteristic frequencies for $k_{x} \propto v_{x}$.

spins, initially oriented in the same direction but following different random trajectories, will be distributed randomly around the Bloch sphere after a relaxation time, $\tau_{s r}$. This relaxation process is known as the D'yakonov-Perel' (DP) mechanism. ${ }^{10}$ It has been demonstrated to be the dominant source of spin relaxation in electron-doped 2DEGs. ${ }^{1,2}$

External magnetic fields have a strong effect on DP relaxation. These effects can be quite complicated when both orbital and spin effects are included. In this paper we discuss only the case of in-plane magnetic fields, which give rise to Zeeman splitting but not to Landau quantization or cyclotron motion. When $\vec{B}_{s o}$ is added to an in-plane magnetic field, $\vec{B}_{\text {ext }}$, it is the total effective field, $\vec{B}_{t o t}=\vec{B}_{s o}+\vec{B}_{\text {ext }}$, that sets the spin-precession axis and precession time for DP spin dynamics. The relaxation time, $\tau_{s r}$, due to the DP mechanism has been calculated ${ }^{11}$ for disordered 2D systems with momentum scattering time $\tau_{p}$, giving

$$
\tau_{s r}\left(B_{e x t}\right) \approx \frac{\tau_{s o}^{2}}{\tau_{p}}\left[1+\left(\tau_{p} \frac{g \mu_{B} B_{e x t}}{\hbar}\right)^{2}\right] .
$$

Here $\tau_{p}$ corresponds to a mean free path $\lambda=\tau_{p} v_{F}$.

The monotonic dependence of $\tau_{s r}\left(B_{\text {ext }}\right)$ described by Eq. (3) does not hold in confined geometries, such as the channels studied here, where the mean free path and spin-orbit length are on the order of or greater than the channel width. ${ }^{12}$ It is the goal of this work to study $\tau_{s r}\left(B_{e x t}\right)$ numerically in these cases.

\section{MODEL}

Semiclassical Monte Carlo simulations of DP spin dynamics in a 2DEG channel were performed by calculating momentum-dependent spin precession along an ensemble of randomly generated classical trajectories, $\vec{r}_{i}(t)$, analogous to the calculations described in Refs. 11, 13, and 14. Spin precession in the total magnetic field along each trajectory was calculated in order to extract the ensemble-averaged spinrelaxation time.

First, an ensemble of trajectories, $\vec{r}_{i}(t)$, was generated to reflect the geometry of the devices used in Refs. 5 and 15: the trajectories were confined to channels that were $1 \mu \mathrm{m}$ wide in the $y$ direction [Fig. 1(a)] but with no boundary in the $x$ direction. The choice of channel orientation along $\hat{x}$ was motivated by Refs. 5 and 15. As can be seen in Eq. (2), $x$ and $y$ coordinates are identical except for a sign reversal so the channel geometry can be effectively rotated by $90^{\circ} \mathrm{sim}-$ ply by reversing the sign of one of the spin-orbit parameters. BSR in arbitrary channel orientations would depend on more complicated combinations of $\alpha$ and $\beta$, but at a qualitative level the observed effects would not change.

The trajectories did not have a fixed length along the channel; rather, the spins bounced in the channel for a fixed time, which is convenient for calculating $\tau_{s r}$. Each trajectory started from the middle of the channel with a random initial velocity direction. It was confirmed that initial conditions had no effect on the calculated spin-relaxation times after averaging over an ensemble of trajectories. Disorder was taken into account in the trajectories primarily through small-angle scattering - the type of disorder that best describes devices in Refs. 5 and 15. Other models of disorder were considered (see Fig. 5), including large-angle scattering and deviations from specularity in channel wall reflections, but no qualitative differences in spin dynamics for different disorder models were found in the range of simulation parameters relevant to experiments. The effect of soft-wall confinement potential due to electrostatic gates was also included.

Each spin $\vec{s}_{i}$ was initialized to lie along the external field, $\vec{s}_{i}(t=0) \| \vec{B}_{\text {ext }}$, as is the case in experiments ${ }^{5,15}$ where spins are injected through quantum point contacts. The spins evolved in time by precessing around the trajectory-dependent $\vec{B}_{\text {tot }}(t)$, calculated using Eq. (2) and $\vec{k}_{i}(t)$. Instantaneous velocity was determined from $\vec{v}_{i}(t)=d \vec{r}_{i} / d t$, giving momentum 
$\hbar \vec{k}_{i}(t)=m^{*} \vec{v}_{i}(t)$ for effective mass $m^{*}$. Throughout this paper the magnitude of the velocity was $\left|\vec{v}_{i}\right|=v_{F}=10^{5} \mathrm{~m} / \mathrm{s}$, corresponding to electron sheet density $n_{s} \approx 5 \times 10^{10} \mathrm{~cm}^{-2}$ in GaAs. ${ }^{5,15}$ The ensemble-averaged projection of the spin on the initial axis was then calculated as a function of time, $P(t)=\left\langle\vec{s}_{i}(t) \cdot \vec{s}_{i}(0)\right\rangle_{i}$, and fit to an exponential decay model, $P(t)=P_{0} e^{-t / \tau_{s r}}$, to extract the spin-relaxation time $\tau_{s r}$.

The semiclassical approximation used in this simulation-classical trajectories with coherent spin precession-is valid when both the orbital phase coherence time and the momentum-scattering time are shorter than the spin-relaxation time, and when electron trajectories can be assumed to be independent of spin direction. The latter criterion implies that the Fermi energy is much larger than the spin-orbit energy, $H_{s o}$. This is a valid approximation in $n$-type GaAs quantum wells but not in $p$-type samples or narrow-gap semiconductors. ${ }^{7}$

\section{RESULTS}

In order to understand BSR, it is important first to consider in detail the types of trajectories that could be expected in a narrow channel of high-mobility 2DEG. Figure 1(a) shows a short segment of a trajectory defined by small-angle scattering with mean free path of $\lambda=10 \mu \mathrm{m}$; this level of disorder is experimentally accessible in high-mobility electron gases. The qualitatively different characteristics of the $x$ and $y$ components of momentum [Figs. 1(b) and 1(c)] highlight the importance of device geometry in the low-disorder regime studied here. Electrons bounce off the channel walls many times before their momentum is randomized, because the mean free path is much larger than the channel width and scattering from the walls is specular.

The trajectories in such a system are characterized by rapid, nearly periodic changes in the sign of the momentum transverse to the channel, $k_{y}$, while the magnitude of the longitudinal momentum, $k_{x}$, changes only diffusively over a longer time scale. The square-wave character of $k_{y}(t)$ can be seen in Fig. 1(c), and in its power spectrum $S_{k y}(f)$ [Fig. 1(d)]. Notice that $S_{k y}(f)$ is strongly peaked despite the random angles of electron motion in a typical trajectory [Fig. 1(a)]. The peak frequency in $S_{k y}(f)$ reflects an average, over the random distribution of trajectory angles, of the bouncing frequency $\frac{v_{F} \sin (\theta)}{2 w}$ for channel width $w$ and angle $\theta$ defined with respect to the $x$ axis.

Relaxation of spins that are aligned initially along $\vec{B}_{e x t}$ results from fluctuating fields transverse to $\vec{B}_{\text {ext }}$. In the DP mechanism, those transverse fields are the momentumdependent effective fields arising from spin-orbit interaction. The first-order component of the effective magnetic field due to $k_{y}$ is always in the $x$ direction [Eq. (2)], independent of the relative strength or signs of Rashba and Dresselhaus terms in the spin-orbit interaction. Similarly, the effective field due to $k_{x}$ is always in the $y$ direction. Hence spins in an external field along $\hat{x}$ relax due to fluctuations in the motion along $\hat{x}$; spins in a field along $\hat{y}$ relax due to fluctuations in the motion along $\hat{y}$.

The qualitatively different spectral characteristics of $x$ and $y$ components of the momentum, $S_{k x}(f)$ and $S_{k y}(f)$, result in

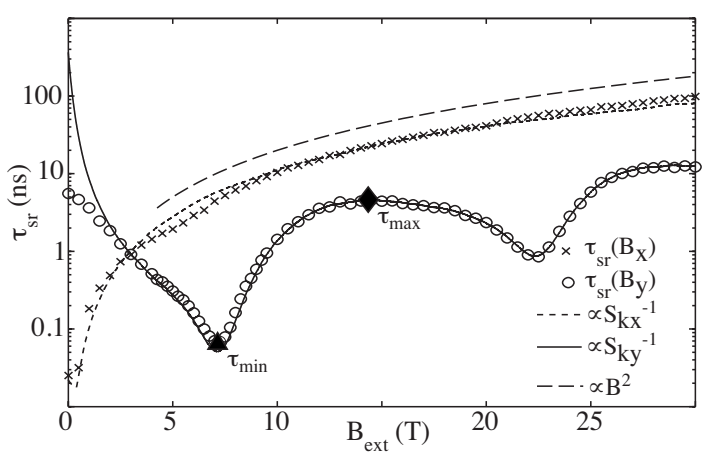

FIG. 2. Spin-relaxation times for $x$ - (crosses) and $y$ - (circles) oriented magnetic fields, for trajectories with the same disorder parameters as in Fig. $1, \alpha=3 \mathrm{meV} \AA$ and $\beta=\gamma=0$ (see text). Solid and short dashed lines are proportional to $S_{k x, y}^{-1}$ as described in the text. Long dashed line shows $B^{2}$ functional form expected in 2D.

qualitatively different relaxation behaviors for spins in $x$ - and $y$-oriented fields. As seen in Fig. 2, $\tau_{s r}\left(B_{x}\right)$ increases smoothly with $B_{x}$ (spins initialized along $\hat{x}$ ), matching the $\tau_{s r} \propto B_{\text {ext }}^{2}$ behavior expected at high field in 2D disordered systems [Eq. (3)] despite the confinement to a micron-wide channel in the simulation. In contrast, $\tau_{s r}\left(B_{y}\right)$ displays sharp periodic dips: the BSR features that are the subject of this paper. The short relaxation time at these dips is spin resonance due to the peak frequencies in $S_{k y}(f)$. This resonance occurs when peaks in $S_{k y}(f)$ occur at the Larmor frequency of the external field, $f_{L}\left(B_{e x t}\right) \equiv g \mu_{B} B_{\text {ext }} / h$. We stress that the difference between $\tau_{s r}\left(B_{x}\right)$ and $\tau_{s r}\left(B_{y}\right)$ is due to the quasi-1D confinement in the channels and is not a consequence of spin-orbit anisotropy. For channels along $\hat{x}, \mathrm{BSR}$ is strongest for $y$-oriented spins even when spin-orbit interaction is isotropic; for channels along $\hat{y}, \mathrm{BSR}$ is strongest for $x$-oriented spins.

The inverse of the spectral densities of the two momentum components, $S_{k}^{-1}(f)$, in Fig. 2 are seen to be directly proportional to the relaxation time extracted from the simulations when $S_{k}(f)$ is evaluated at the Larmor frequency: $\tau_{s r}\left(B_{\text {ext }}\right) \propto S_{k}^{-1}\left(f_{L}\right)$. This is reminiscent of the nuclear spinrelaxation time, $T_{1}$, for nuclear magnetic resonance, for which it has been shown that $T_{1}^{-1}=\left(g \mu_{B} / \hbar\right)^{2} S_{B_{\perp}}\left(f_{L}\right)$ with $S_{B}\left(f_{L}\right)$ representing the spectral density of fluctuations in the transverse magnetic field, $B_{\perp}$, at the Larmor frequency of the static NMR field. ${ }^{16}$ Fluctuations in $B_{s o}$ are proportional to $S_{k}(f)$ by Eq. (2), and it is these fluctuations that lead to relaxation in the present case. The approximation $\tau_{s r}\left(B_{e x t}\right) \propto S_{k}^{-1}\left(f_{L}\right)$ becomes significantly worse when the mean free path is much longer than $10 \mu \mathrm{m}$ (data not shown), perhaps because the assumption of exponentially correlated noise in the NMR result breaks down.

The parameters $\alpha, \beta$, and $\gamma$ in Eq. (1) control both the magnitudes and symmetries of the spin-orbit field. Because $\tau_{s r}\left(B_{y}\right)$ depends on the $x$ component of $\vec{B}_{s o}$, it is controlled by $(\alpha-\beta)$ and is nearly independent of $(\alpha+\beta)$ [Eq. (2)]. The accuracy of this approximation can be tested in the simulation by varying $\alpha$ and $\beta$ independently. As seen in Fig. 3(a), curves with identical $(\alpha-\beta)$ but different $(\alpha+\beta)$ fall on top of each other for $B_{y} \gtrsim 3 \mathrm{~T}$. [The stronger dependence on 

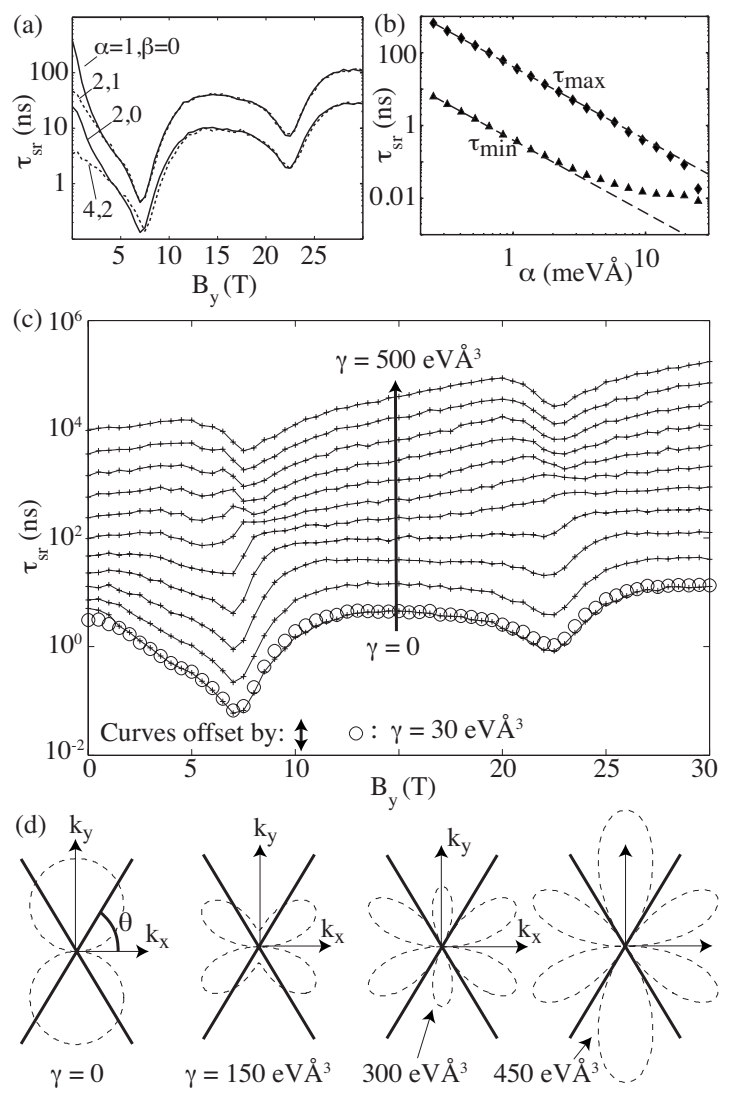

FIG. 3. (a) Relaxation times for different linear spin-orbit coupling strengths, denoted as $(\alpha, \beta)$. At high field the relaxation time $\tau_{s r}\left(B_{y}\right)$ is determined by $(\alpha-\beta)$, while at low fields $\tau_{s r}\left(B_{y}\right)$ also depends significantly on $(\alpha+\beta)$. (b) Relaxation time at the first local minimum $\left(\tau_{\min }, B_{y} \approx 7 \mathrm{~T}\right)$ and local maximum $\left(\tau_{\max }, B_{y} \approx 14.5 \mathrm{~T}\right)$ from Fig. 2, plotted against $\alpha(\beta=\gamma=0)$. Throughout the low-spin-orbit range $(\alpha<6 \mathrm{meV} \AA), \tau_{s r} \propto \alpha^{-2}$ (dashed lines). (c) Effect of third-order spin-orbit term, quantified by $\gamma$, on BSR features; first-order terms fixed at $\alpha=3 \mathrm{meV} \AA$, $\beta=0$. Each solid-line curve represents a different $\gamma \in\{0,50,100, \ldots, 500\}$ eV $\AA^{3}$, vertically offset as shown. Circles represent $\gamma=30 \mathrm{eV} \AA^{3}$ (expected for GaAs 2DEGs) and fall on top of the $\gamma=0$ curve. Panels (a)-(c) use disorder parameters from Fig. 1(d). Polar plots (dashed lines) of the $x$ component of $\vec{B}_{s o}$, $r(\theta)=\left|\vec{B}_{s o}(\theta) \cdot \hat{x}\right|$ for trajectory angle $\theta$. Four graphs show different values of $\gamma$, all with $\alpha=3 \mathrm{meV} \AA, \beta=0$. For comparison, length of bold lines indicates field strength $r=1.6 \mathrm{~T}$ at angle $\theta= \pm 1 \mathrm{rad}$.

$(\alpha+\beta)$ at low field comes about because the direction of $\vec{B}_{t o t}$ fluctuates significantly when $B_{e x t} \leqslant B_{s o}$.] The dependence of $\tau_{s r}$ on $\alpha$ is shown in Fig. 3(b), holding $\beta=0$ for all curves: when examined for particular values of magnetic field, the relation $\tau_{s r} \propto \tau_{s o}^{2} \propto \alpha^{2}$ expected for $2 \mathrm{D}$ [Eq. (3)] carries over to the channel data.

The discussion thus far has ignored the $O\left(k^{3}\right)$ term in Eqs. (1) and (2). The strength of this term is $\frac{2}{\pi} \gamma k_{F}^{3}$ when averaged over the Fermi circle. Using values for $\gamma$ reported in the literature $\left(9-34 \mathrm{eV}^{3}\right)$ (Ref. 8) and 2DEG parameters $v_{F}=1 \times 10^{5} \mathrm{~m} / \mathrm{s}$ and $|\alpha|+|\beta| \approx 3 \mathrm{meV} \AA$ reported in Ref. 5, the third-order spin-orbit field, $B_{s o}^{(3)}$, is an order of magnitude smaller than the first-order field, $B_{s o}^{(1)}$. For this reason, the simulations presented in most of this paper set $\gamma$ explicitly to zero for ease of calculation.

For significantly larger values of $v_{F}$ or $\gamma$, on the other hand, $B_{s o}^{(3)}$ is of the same order or larger than $B_{s o}^{(1)}$. Because of the more complicated symmetry of $B_{s o}^{(3)}$, its effect on BSR is not monotonic in $\gamma$. Figure $3(\mathrm{c})$ explores the role of $B_{s o}^{(3)}$ by raising $\gamma$ while holding $v_{F}=1 \times 10^{5} \mathrm{~m} / \mathrm{s}, \alpha=3 \mathrm{meV} \AA$, and $\beta=0$. Although this particular set of parameters could not be easily achieved in a single sample, where $\beta \approx-\gamma\left\langle k_{001}^{2}\right\rangle$, it provides a clearer distinction between the roles of first- and third-order terms.

The BSR dips disappear around $\gamma \approx 300 \mathrm{eV} \AA^{3}$, then revive for larger values of $\gamma$ [Fig. 3(c)]. The disappearance of the dips in $\tau_{s r}\left(B_{y}\right)$ can be explained by zeros in the transverse (x) component of the total spin-orbit field, $\vec{B}_{s o} \equiv \vec{B}_{s o}^{(1)}+\vec{B}_{s o}^{(3)}$, at trajectory angles corresponding to the Larmor frequency of the dips. These zeros arise due to a cancellation of the firstand third-order terms [Fig. 3(d)]. Considering the first dip, at $B_{y} \approx 7 \mathrm{~T}$, the condition $\frac{v_{F} \sin (\theta)}{2 w}=g \mu_{B} B_{\text {ext }} / h$ implies $\theta \approx \pm 1 \mathrm{rad}$. Figure 3(d) shows the angular dependence of $x$ component of $B_{s o}$ for four different values of $\gamma$. When $\gamma=300 \mathrm{eV} \AA^{3}$, the magnitude is zero for $\theta \approx 1 \mathrm{rad}$ as expected. The spin-resonance dips revive, and shift to higher field, for larger $\gamma$ because the $x$ component of $B_{s o}$ is enhanced near $\theta= \pm \pi / 2$, adding more weight to spin relaxation from trajectories that traverse the channel directly with correspondingly higher bouncing frequencies.

Significant changes in spin relaxation are observed when the overall magnitude of disorder (set by $\lambda$ ) is varied [Fig. 4(a)]. When $\lambda$ is much smaller than the channel width, resonant dips are absent. In that case, the bouncing frequency ceases to be a relevant parameter, as electrons seldom make it across the channel without scattering and the 2D limit of Eq. (3) is approached. The dips become deeper as $\lambda$ is increased, but reach a minimum value around $10 \mu \mathrm{m}$ before rising again for even longer mean free path.

In order to understand this nonmonotonic dependence, we study the $\lambda$ dependence of $\tau_{s r}$ at the first resonant dip, around $B_{y}=7 \mathrm{~T}$ [Fig. 4(b)]. Starting from very short mean free paths, $\tau_{s r}$ reaches a minimum at $\lambda_{\min }$, then rises again for very long mean free paths. The length scale, $\lambda_{\min }$, corresponds to $2 \pi v_{F} \tau_{s o}$, the distance an electron would have to travel in order for the spin to rotate by $2 \pi$ due to the spinorbit effective field.

This behavior can be explained at a qualitative level by considering spin relaxation in a reference frame that rotates at the Larmor frequency. Working in this frame effectively removes precession due the external field, and it removes flips in $\vec{B}_{s o}$ that occur at frequency $f_{L}$ due to bouncing between the channel walls. In other words, spin relaxation in the ballistic channel at the BSR condition is approximately mapped onto spin relaxation in a disordered 2D system at zero external magnetic field. In 2D at zero external field, one expects $\tau_{s r} \approx \tau_{s o}^{2} / \tau_{p} \equiv \tau_{s o}^{2} v_{F} / \lambda$ [Eq. (3)] to decrease with increasing $\lambda$ in the motional narrowing regime, i.e., for fast momentum relaxation, $\tau_{p}<\tau_{s o}$. In the ballistic limit, $\tau_{p}>\tau_{s o}$, on the other hand, one expects $\tau_{s r}$ to increase with $\lambda$ as $\tau_{s r} \approx \tau_{p}=\lambda / v_{F}$ because spins precess coherently between scattering. ${ }^{11,13}$ It is the crossover from motional narrowing to 


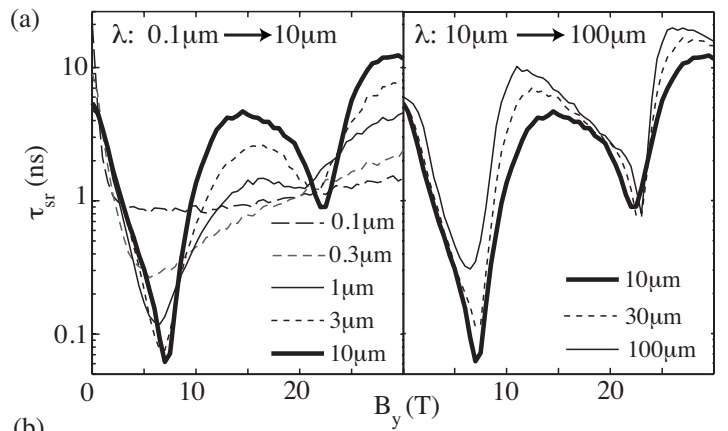

(b)

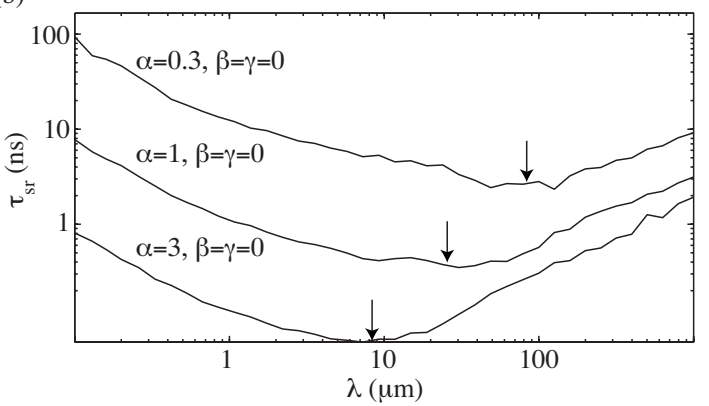

FIG. 4. (a) Relaxation times for different mean free paths in a 1 - $\mu \mathrm{m}$-wide channel. Left- and right-hand panels show mean free paths less than and greater than $10 \mu \mathrm{m}$, respectively, with the $\lambda=10 \mu \mathrm{m}$ trace appearing in both panels. Signature of ballistic spin resonance is clearly visible even when $\lambda$ is as short as the channel width $1 \mu \mathrm{m}$. Features continue to sharpen (including faster spin relaxation at resonance) up to $\lambda=10 \mu \mathrm{m}$. For longer mean free paths, relaxation time at resonance rises again. (b) Effect of mean free path on relaxation time at the first local minimum $\left(\tau_{\min }, B_{y} \approx 7 \mathrm{~T}\right)$ in a $1-\mu \mathrm{m}$-wide channel. Arrows denote the crossover value $\lambda_{\min }=2 \pi v_{F} \tau_{s o}$ discussed in text.

ballistic regimes that gives rise to the nonmonotonic behavior of $\tau_{s r}$ in Fig. 4(b).

Finally, we show that the particular type of disorder used to generate trajectories, and the type of scattering off channel walls, has only a small effect on the simulated spinrelaxation curves. Figure 5 shows spin relaxation for three different types of disorder.

(1) Small-angle scattering is believed to be the dominant scattering mechanism in high-mobility GaAs 2DEGs. ${ }^{17,18}$ This was modeled by changing the direction of motion from time step to time step by a small random angle that was Gaussian distributed around zero with standard deviation calculated to give the desired mean free path.

(2) Large-angle scattering was implemented as a probability for complete randomization of momentum angle at each time step. The probability was calculated to give the desired mean free path.

(3) Rough potential walls. Upon reflection off channel walls, the angle of reflection was randomly distributed around the angle of incidence with a spread of $\phi_{\text {spec }} . \phi_{\text {spec }}=0$ corresponds to specular scattering from channel walls. This effect is believed to be weak in electrostatically defined GaAs 2DEG nanostructures, as shown by clear transverse focusing signals even up to high order, which requires many specular bounces. ${ }^{19}$

Each curve in Fig. 5(b) corresponds to disorder from only one of the three mechanisms. The mean free path is
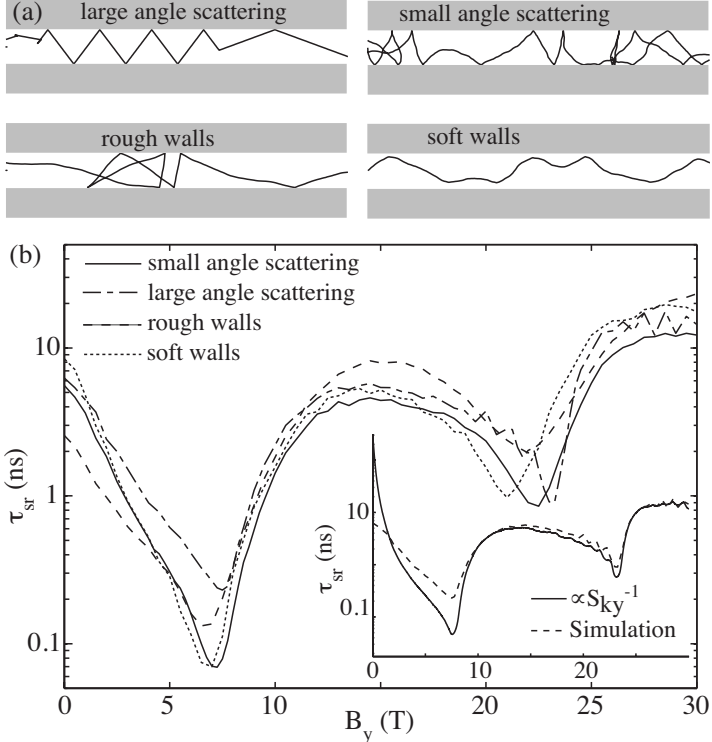

FIG. 5. (a) Cartoon trajectories corresponding to the types of disorder and scattering described in the text. (b) Spin-relaxation time for $B_{\text {ext }} \| \hat{y}$, calculated for four disorder models, each giving effective $\lambda=10 \mu \mathrm{m}$. Solid: small angle scattering. Dashed-dotted: large angle scattering. Short dashed: rough walls (nonspecular scattering), plus small angle scattering giving $\lambda=100 \mu \mathrm{m}$ for trajectories that would otherwise not encounter a wall. Dotted: small angle scattering and soft walls, modeled by a carrier density that decreases smoothly to zero within $\lambda_{d e p}=150 \mathrm{~nm}$ of channel walls. Inset: match between the power spectral density and spin-relaxation time is much worse in the case of large angle scattering, compared to small angle scattering [see, e.g., Fig. 2(a)] and other disorder models (data not shown) $(\alpha=3 \mathrm{meV} \AA, \beta=\gamma=0)$.

$\lambda=10 \mu \mathrm{m}$ in each case, confirmed by monitoring the autocorrelation of $k_{x}(t)$. As seen in the figure, the simulated spinrelaxation time depends only slightly on the precise model of disorder, despite the importance of ballistic transport to the resonant dips in $\tau_{s r}\left(B_{y}\right)$. One qualitative difference between disorder models was the match between $\tau_{s r}\left(B_{y}\right)$ and $S_{k y}^{-1}(f)$, which was significantly worse for large angle scattering compared to the other two [compare, for example, inset of Fig. 5(b) to Fig. 2]. Although Fig. 5 focuses on $\lambda=10 \mu \mathrm{m}$, similar conclusions were drawn over a wide range of mean free paths (data not shown).

Figure 5(b) also compares BSR for the case of simple reflections from hard-wall channel boundaries to the more realistic case of soft walls with a $150 \mathrm{~nm}$ depletion length as might be expected in nanostructures defined by electrostatic surface gates. Small-angle scattering is implemented to give $\lambda=10 \mu \mathrm{m}$ in both hard-and soft-wall simulations. The difference between the hard- and soft-wall data is nearly indistinguishable, except for a small shift in the field at which the resonance dips occur.

\section{CONCLUSIONS}

Ballistic spin resonance in quasi-1D channels of twodimensional electron gas was studied numerically using semiclassical Monte Carlo simulations of spin dynamics due 
to the D'yakonov-Perel' mechanism. The main conclusions of the paper are: (1) BSR is a robust effect that can be expected to occur over a wide range of disorder and spin-orbit strength in a real 2DEG.

(2) BSR occurs when external magnetic field is applied in the 2DEG plane perpendicular to the channel axis. The size of the BSR dip depends on $\alpha-\beta$ in channels fabricated along [110] and $\alpha+\beta$ in channels along [110]. The condition $\alpha=\beta$, for example, would correspond to a complete disappearance of BSR for [110] channels. $^{20}$ This demonstrates the value of measuring BSR for both [110] and [110] channel orientations, as a strategy for determining $\alpha$ and $\beta$ independently.

The simulated $\tau_{s r}\left(B_{y}\right)$ can be compared to the experimentally measured field dependence of spin-relaxation length in
2DEG channels in order to extract spin-orbit parameters. ${ }^{5}$ The values of electron density and mean free path required for the simulations can be obtained from charge-transport measurements. It should be noted that experiments performed so far did not access spin-relaxation time directly. Instead they measured spin accumulation set by diffusion. Therefore comparison of D'yakonov-Perel' spin dynamics to experiments required a substitution of simulated spinrelaxation times into a diffusion equation. ${ }^{5}$

\section{ACKNOWLEDGMENTS}

The authors thank J. C. Egues, M. Lundeberg, and G. Usaj for valuable discussions. Work at UBC was supported by NSERC, CFI, and CIFAR.
${ }^{1}$ G. E. Pikus and A. N. Titkov, in Optical Orientation, edited by F. Meier and B. P. Zakharchenya (Elsevier, New York, 1984), p. 73.

${ }^{2}$ I. Žutić, J. Fabian, and S. Das Sarma, Rev. Mod. Phys. 76, 323 (2004).

${ }^{3}$ J. H. Bardarson, I. Adagideli, and P. Jacquod, Phys. Rev. Lett. 98, 196601 (2007).

${ }^{4}$ S. Datta and B. Das, Appl. Phys. Lett. 56, 665 (1990).

${ }^{5}$ S. M. Frolov, S. Lüscher, W. Yu, Y. Ren, J. A. Folk, and W. Wegscheider, Nature (London) 458, 868 (2009).

${ }^{6}$ H. C. Koo, J. H. Kwon, J. Eom, J. Chang, S. H. Han, and M. Johnson, Science 325, 1515 (2009).

${ }^{7}$ R. Winkler, Spin-Orbit Coupling Effects in Two-Dimensional Electron and Hole Systems, Springer Tracts in Modern Physics Vol. 191 (Springer, Berlin, 2003).

${ }^{8}$ J. J. Krich and B. I. Halperin, Phys. Rev. Lett. 98, 226802 (2007).

${ }^{9}$ J. B. Miller, D. M. Zumbühl, C. M. Marcus, Y. B. LyandaGeller, D. Goldhaber-Gordon, K. Campman, and A. C. Gossard, Phys. Rev. Lett. 90, 076807 (2003).

${ }^{10}$ M. I. D'yakonov and V. I. Perel', Sov. Phys. Solid State 13, 3023 (1972).
${ }^{11}$ A. A. Kiselev and K. W. Kim, Phys. Rev. B 61, 13115 (2000).

${ }^{12}$ A. W. Holleitner, V. Sih, R. C. Myers, A. C. Gossard, and D. D. Awschalom, Phys. Rev. Lett. 97, 036805 (2006).

${ }^{13}$ E. Koop, B. van Wees, and C. van der Wal, arXiv:0804.2968 (unpublished).

${ }^{14}$ J. Liu, T. Last, E. J. Koop, S. Denega, B. J. van Wees, and C. H. van der Wal, J. Supercond. Novel Magn. 23, 11 (2010).

${ }^{15}$ S. M. Frolov, A. Venkatesan, W. Yu, J. A. Folk, and W. Wegscheider, Phys. Rev. Lett. 102, 116802 (2009).

${ }^{16}$ C. P. Slichter, Principles of Magnetic Resonance (Harper \& Row, New York, 1963).

${ }^{17}$ P. T. Coleridge, Phys. Rev. B 44, 3793 (1991).

${ }^{18}$ M. P. Jura, M. A. Topinka, L. Urban, A. Yazdani, H. Shtrikman, L. N. Pfeiffer, K. W. West, and D. Goldhaber-Gordon, Nat. Phys. 3, 841 (2007).

${ }^{19}$ H. van Houten, C. W. J. Beenakker, J. G. Williamson, M. E. I. Broekaart, P. H. M. van Loosdrecht, B. J. van Wees, J. E. Mooij, C. T. Foxon, and J. J. Harris, Phys. Rev. B 39, 8556 (1989).

${ }^{20}$ J. D. Koralek, C. P. Weber, J. Orenstein, B. A. Bernevig, S. Zhang, S. Mack, and D. D. Awschalom, Nature (London) 458, 610 (2009). 